\title{
Forced Response Vibration Analysis of the Turbine Blade with Coupling between the Normal and Tangential Direction
}

\author{
Aram Mahmoodi $(\mathbb{D}$ and Hamid Ahmadian \\ Center of Excellence in Experimental Solid Mechanics and Dynamics, School of Mechanical Engineering, \\ Iran University of Science and Technology, Narmak, Tehran 16844, Iran \\ Correspondence should be addressed to Aram Mahmoodi; mahmoodiaram@gmail.com
}

Received 1 October 2021; Revised 16 December 2021; Accepted 5 January 2022; Published 4 February 2022

Academic Editor: Dario Di Maio

Copyright (c) 2022 Aram Mahmoodi and Hamid Ahmadian. This is an open access article distributed under the Creative Commons Attribution License, which permits unrestricted use, distribution, and reproduction in any medium, provided the original work is properly cited.

\begin{abstract}
This paper is about the nonlinear dynamic of turbine blades due to the friction between blades. This paper investigates the coupling effect in an experimental turbine blade model. In this contact model, coupling between friction in tangential and normal directions and energy dissipation in the lateral direction are considered simultaneously, which has not been considered elsewhere in the contact model of the turbine blade. The mathematical model of the blade under-platform damper model is derived, and to solve nonlinear equations, the multi-harmonic balance method is used. The contact force is calculated by the alternative frequency time-domain method in this solution method. To solve nonlinear derived algebraic equations, a continuation algorithm is applied. It is shown that considering the coupling phenomenon causes the results to be different from the situation in which this physical phenomenon is ignored. To validate the algorithm of the solution, numerical results of previous references in the turbine-blade field are regenerated. Experimental analysis on the under-platform damper and turbine blade model is done to investigate the coupling effect. It is shown that the contact model with consideration with coupling effect between tangential and normal direction can predict experimental results (amplitude and frequency of resonance) most of the other contact models used in the turbine field. To accurately determine the amplitude and frequency of resonance, it is necessary to consider the coupling effect.
\end{abstract}

\section{Introduction}

The blade disk is one of the essential components in turbine machinery. It transfers energy from the gas flow going through turbo-machinery. In turbine blades, avoiding resonance is almost impossible because the frequency band of force excitation and natural frequencies of the blade disk are broadband ranges and overlap ranges $[1,2]$. Forced vibration analysis of bladed disk assemblies is a significant part of the design of gas turbine engines. One of the main problems of the gas turbine engines is the high cycle fatigue failure of turbine and compressor blades due to the blade vibration resonance in the operating range [3]. Preventing turbomachinery blade failures is essential for the gas turbine engine developers, and therefore, avoiding excessive vibration in turbo-machinery blading is crucial [1]. To obtain this goal, using dry friction dampers (like a shroud and under-platform damper) in the turbo-machinery industry is very common.

Vibration analysis of the turbine blade with dry friction damper due to the contact is nonlinear. Most of the research works in the contact modeling turbine blade vibration are about to model the friction in the contact interface $[1,4,5]$. The literature review used different models to model contact force and forced response $[6,7]$. Griffen used the model that displacement of contact is limited to the contact surface, and normal displacement is neglected [6]. Menq et al. [7] Used model that considers micro slip displacement in forced response analysis of fan with shroud. They also compared their analysis with experimental results. A friction model with normal load variation has been presented by Yang et al. [8]. This model uses linear variation in the normal direction and Coulomb's law in the tangential direction to model dry friction force. Petrove and Ewins published their work for 
one-dimensional tangential motion and normal load variation like the Yang model [9] in 2003. It is worth considering that applied this model in practical turbine blade model due to the calculation of Jacobian by finite difference increases computational time to convergence response. Analytical Jacobian of contact force has been presented by reference [10] to solve this problem.

Contact in the normal direction in turbine blade analysis is usually considered as stable contact, although in the literature review there are several references $[11,12]$ that consider this hysteresis effect and its dissipation energy by compensation coefficient or by viscous finite element [5].

In reference [13] for modeling friction contact in the shroud blade, the unilateral spring with a gap is used to model nonlinearity contact in the normal direction. The results show that increasing the damper gap size causes an increase in resonant response. Also, in references $[14,15]$ to model the shroud contact, researchers only consider the impact effect on frequency response function on the blade.

Pennacchi and et al. [16] investigated friction and impact (called snubbing effect) simultaneously in a turbine blade. They calculated the vibration of the turbine blade by numerical integration and then reducing the vibration level due to the snubbing effect is showed. They also show that combination parameters of the system and excitation force in some frequencies can increase frequency response, not resonance frequencies.

One of the big challenges in accurately modeling contact surfaces is considering the normal lift-up due to the tangential motion because all surfaces have the roughness that causes this motion (as a result, moving in a tangential direction causes a change in normal force). The literature review survey about contact models used to simulate contact force in the blade-turbine field shows that none of them considered this effect in their simulation, while the importance of this effect to accurately predict contact force has recently been demonstrated in references [17]. In this study, the effect of coupling between the normal and tangential direction to simulation contact force in the nonlinear surface and its effect on predicting the turbine blade's response is investigated. By comparison, theoretical results with experimental analysis show that ignoring the coupling effect between two directions causes an error in predicting the response and considering its effect to precise prediction is essential.

For solving nonlinear equations in turbine blades vibration analysis, three different approaches are used in the literature: the analytical approach, time-domain method, and frequency domain simulation by using multiharmonic balance $(\mathrm{MHB})$ method. The analytical approach is applicable in simple models, including one and two DOF $[18,19]$. In the time-domain approach, nonlinear equations of motion are integrated numerically [8, 20, 21]; timedomain integration can be implemented easily. However, steady-state solutions can be obtained through transient solutions, which increase the computational time considerably, and due to this long computational time rarely used in practical problems, it is used to validate other numerical methods.
The third approach, the MHB method, is widely used to compute forced response analysis in the blade disk vibration. In this method, excitation, nonlinear force, and the system's response are represented by Fourier series approximation. This method is so common in the calculation of forced response analysis of frictionally damped bladed disks [22-25]. Using the Harmonic balance method and the alternative frequency-domain method (HB-AFT method) Creates a robust method that other general analytical methods such as multiple-scale failed to give analytical method can use to calculate analytical solution [26]. The HBAFT method with arc-length continuation to trace branches of nonlinearity in turbine blade vibration analysis is more applicable [27, 28].

The experimental test in the friction damping field in turbine blade due to the nonlinear nature of contact has an important role. Validation of numerical solution and predicting contact parameters of the contact surface is the main objective of the experimental test. Gastaldi et al. [29] designed a new test rig to identify contact parameters of the under-platform damper. They used this study for sensitivity analysis of under-platform's contact parameters. In reference [30], dynamic behavior of under-platform with a setup including a blade and two under-platform dampers are investigated. They extracted a hysteresis loop for the corresponding point of FRF that by this set of diagrams can explain unexpected phenomena in the experimental test.

Ferhatoglu et al. [31, 32] present a numerical method to capture multiple responses in a turbine with an underplatform damper. Due to the uncertainty phenomena, a dry friction calculation worse case of response from a design point of view is essential. Recently some improvement to enhance the efficiency of under-platform damper, like adding piezoelectric material to the flexible damper, has been published to open a new research field on friction damper in the turbine blade industry [33].

In friction damper design, the geometry of the underplatform damper and contact surface are important parameters to optimize the efficiency of dampers. Different studies [34-36] in the literature investigated this parameter on under-platform dampers. Panning and et al. [35] showed that breaking the symmetry increases the damping effect and under-platform geometry has a remarkable effect on damping efficiency.

In the present paper, nonlinear dynamic of turbine blades with considering dissipation energy in the tangential direction in the lateral motion is investigated. The innovation of this paper related to other works in the field of turbine blade vibration is to consider the effect of tangential displacement on normal load variation (coupling between two directions-lift-up phenomena). The MHB method is used to convert the time-domain equation to the frequency domain, and the alternative frequency time (AFT) domain strategy is applied to calculate harmonics of nonlinear force in the frequency domain. To solve the algebraic equation and to catch turning points (in this case resonance frequency variation), the continuation method is used [36]. To validate the effectiveness and stability of the algorithm, the results of the common model applied in turbine blade contact in 
reference [37] are regenerated. To investigate the coupling effect on frequency response function, the experimental analysis on the model of the turbine blade is done. In the experimental analysis, the coupling effect on the frequency response of the system has been investigated and its effect on the precise determination of design parameters (amplitude and frequencies of resonance) has been studied.

\section{Forced Vibration of the System}

Force vibration of each nonlinear system has an equation of motion according to the following equation [38]:

$$
M \ddot{x}+C \dot{x}+K x=f_{n}(x, \dot{x}, t)+f_{\text {exc }}(t),
$$

where $M, C$, and $K$ stand for the mass, viscous damping, and linear stiffness matrices, respectively. $x, \dot{x}, \ddot{x}$ are displacement vector and its derivatives, respectively. $f_{n}(x, \dot{x}, t)$ is the term of internal nonlinear force that is explained more in the nonlinear force subsection. The gas flow is disturbed at the nonrotating parts and rotating parts when it flows through the gas intake and usually forms a harmonic exciting force on the blades. Therefore, the exciting force on the blades is often described as the harmonic force [34]:

$$
f_{\text {exc }}(t)=A \exp \left(\omega t+\varphi_{j}\right), \quad \varphi_{j}=\frac{2(j-1) \pi E}{N} .
$$

Here, $A$ is force amplitude, $\omega$ is excitation frequency and $E$ is engine order (in a turbo-machine flow path, there are several cases where there is some finite number of flow distortions upstream or downstream of a component such as a turbine bladed-disk. The number of distortion is called engine order), $j$ is the number of the blade where excitation is applied, and $N$ is the number of whole blades.

The main challenge in the solution of nonlinear equations in turbine-blade is to calculate contact force. As mentioned in the introduction, there are different models to simulate this contact force, but the main model is the Menq model or a simple form of it $[1,5,10,34,39,40]$. The most significant drawbacks of these models are to neglect the effect of tangential displacement on normal force and ignore energy dissipation in the normal direction. While in practical contact surfaces like under-platform damper and shroud, these effects are considerable, and in shroud contact, impact dissipation is inevitable. Recently, the importance of coupling effect to accurately simulate the nonlinear force of contact surface has been demonstrated by Jamshidi et al. [17].

The innovation of this paper is to consider this effect and increased frequency response function precision since the precise determination of resonance point and vibration amplitude is essential in determining contact parameters to optimum dry friction efficiency. Using the model provided by MohammadAli et al. [2], precisian simulation from contact force was provided in this study. In this model, the normal force and tangential force variation are expressed by the following relations [2]:

$$
\begin{aligned}
& f_{x}=C_{Q} f_{y}^{m} \Delta_{T}, \\
& \Delta_{T}=|\Omega| \operatorname{sgn}(x)\left(1-\exp \left(\frac{n C_{k} C_{P}{ }^{1-m}}{C_{Q} w^{1-n(1-m)}} \operatorname{sgn}(x)\left(x_{0}-x\right)\right)\right)+\Delta_{T_{0}} . \\
& f_{y}=C_{p} y^{n} \Delta_{P}, \Delta_{P}=\Delta_{N}+\left(1-\Delta_{N}\right)\left|\Delta_{T}\right|, \\
& \Delta_{N}= \begin{cases}1, & 0<y, \quad \text { Loading, } \\
\exp \left(-k_{N}\left(y_{\max }-y\right)\right), & 0<y \leq y_{\max }, \quad \text { Unloading, } \\
0, & 0 \geq y, \quad \text { Separation. }\end{cases}
\end{aligned}
$$

That, $C_{p}, n, K_{N}, C_{Q}, m, C_{k}, C_{p}, n$ are constant parameters and $f_{x}, f_{y}, x, y, y_{\max }$ are tangential force, normal force, friction displacement, normal displacement, and maximum displacement in the normal direction, respectively. For more information about this model and its parameters readers can study reference [2].

Equation (4) considers the effect of tangential displacement on normal force. In the following section, the effect of this term on the frequency response function in the experimental test was investigated.

It is worth mentioning that these relations expressed nonlinear force in the time domain, while frequency descriptions are used in the harmonic balance method. To solve this problem, the AFT method is used. This method is explained in Figure 1 that readers for more information can refer to references [38, 41-43]:

The MHB method to solve the nonlinear equation method is used. In this method (MHB) where the excitation force is harmonic, the response is assumed according to the following equation [36].

$$
q(t)=Q_{0}+\sum_{j=1}^{n} Q_{2 j-1} \cos \left(m_{j} \omega t\right)+Q_{2 j} \sin \left(m_{j} \omega t\right),
$$

where $Q_{j}(j=0 \ldots 2 n)$ are harmonic balance coefficients for degrees of freedom of system (DOF), $m_{j}(j=1 . . n)$ is determined the harmonic number and $\omega$ is the primary frequency of excitation. After substation equation (5) in (1) and 


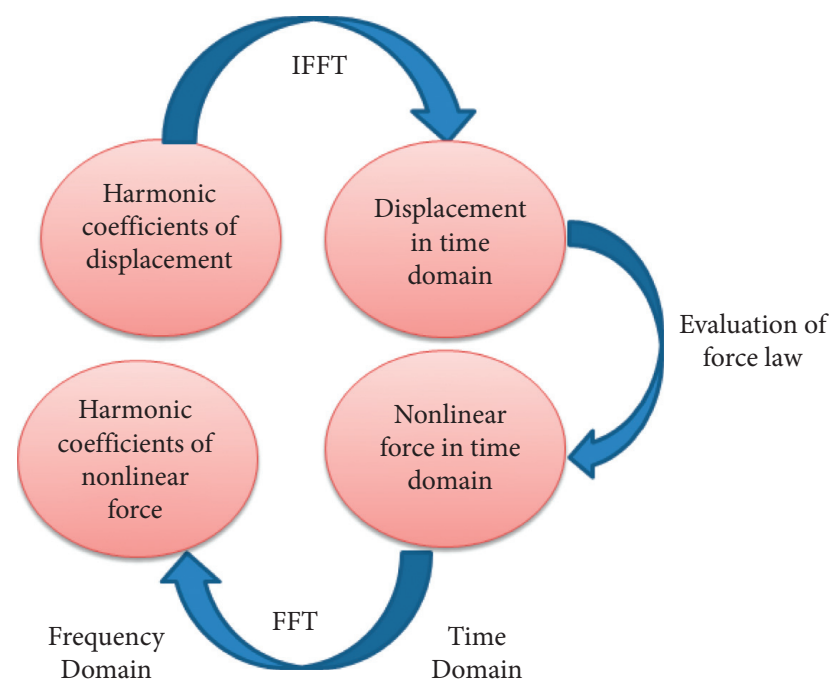

Figure 1: AFT description to calculate the nonlinear force [36].

applied Galerkin method [36] final form of algebraic equation in the frequency domain is according to the following equation:

$$
R(Q)=Z(\omega) Q+F(Q)-P=0,
$$

where $Q=\left\{Q_{0}, Q_{1}, Q_{2}, \ldots, Q_{2 n-1}, Q_{2 n}\right\}$ is a harmonic vector, $F(Q)$ is nonlinear force, and $P$ is the harmonic vector of excitation force.

To solve algebraic equations in this paper, the Newton-Raphson method with the continuation algorithm were used. In the Newton-Raphson method, response of the algebraic equation was calculated according to the following iteration:

$$
Q^{(k+1)}=Q^{(k)}-\left(Z(\omega)+\frac{\partial F\left(Q^{k}\right)}{\partial Q^{k}}\right)^{-1}\left(R\left(Q^{(k)}\right)\right) .
$$

In equation (7), $\partial F\left(Q^{k}\right) / \partial Q^{k}, F\left(Q^{k}\right)$ are Jacobin of nonlinear force related to nonlinear displacement and nonlinear force, respectively. One of the big challenges in the solution method is the calculation of these terms. Different contact models were used in the literature to express nonlinear force, but it is too difficult to find a model with both precision and explicit form simultaneously. This paper uses a model that simulates nonlinear force in the normal direction and tangential ones simultaneously. The main innovation of this paper is to consider tangential displacement on the calculation of normal force that this effect is considered in modeling nonlinear force in the contact surface [17].

Due to the nonlinearity in equation (1) just using the numerical solution, (5), finding a turning point in the solution is impossible. To solve this problem in this paper, the continuation method [36] was used. In the continuation method, there are three steps to find the turning point (that in frequency response function, output results of this paper, is resonance frequency shift due to the nonlinear force): (1) Predictor (2) Parameterization (3) Corrector step. Describe the continuation method beyond this paper's scope for more information readers can refer to [44].
For verification of the solution method, the results regenerated by this study are compared with the results of the following reference [40]. In reference [40], Joannin et al. simulated nonlinear vibration of turbine blade by below model (they model each blade by four Degree of freedom and consider six blades in their study (Figure 2)).

The tan-hyperbolic function was used to model contact force:

$$
f_{n l}(\dot{x})=\mu N \tanh \left(\frac{\dot{x}_{r e l}}{\varepsilon}\right),
$$

where $\dot{x}_{r e l}$ is the relative displacement of contact in the tangential direction. $\mu$ and $N$ are constant parameters representing friction coefficient and normal load in the contact interface, respectively. $\varepsilon$ is regularization level, a smaller value of $\varepsilon$, better the approximation of sign function but longer convergence time [40]. It is worth mentioning that this model is an explicit form of a common model that has been used to simulate contact force in nonlinear turbineblade $[5,37]$. For more information about the model and its parameters, reader can refers to [40].

In this study, to solve the nonlinear equation, the MHB method with AFT is used. To validate the solution of this paper, the frequency response function turbine blade model in Figure 3 was extracted.

As shown in Figure 3, the result is correlated. From the results of Figure 3, it is concluded that the algorithm of the solution was used in this paper is valid and could use to predict the results of the practical turbine blade model. In this section, the algorithm of the solution was validated. In Section 3, using the experimental test and the solution method of this section, the coupling effect in a turbine blade model is investigated.

\section{Experimental Analysis}

In this section, the effect of coupling between normal and tangential direction by experimental analysis is investigated. The efficiency prediction of using the model to simulate nonlinear contact force is validated. The experimental sample shown in Figure 4 is made of two steel beams that represent two turbine blades and are held in place by a base. A similar laboratory sample is used in references $[24,45,46]$.

In a similar experiment, the laboratory sample was bound to a large rigid body, but in this study, the test in free position is done (Figure 5). The centrifugal force is simulated using the spring force. The spring has an initial deformation of one centimeter, and due to the stiffness of the spring, it has provided a normal force equal to $135 \mathrm{~N}$. The stiffness of the spring is calculated using a static test of $13.5 \mathrm{~N} / \mathrm{m}$. In Figure 6, the laboratory sample size is shown.

3.1. Linear Analysis. To investigate overall dynamic response of the systems, linear analysis of blades without underplatform damper is done.

The structure is excited by a shaker at the junction between two blades with random and sine sweep signals excitation. Random excitation is used to determine the linear 


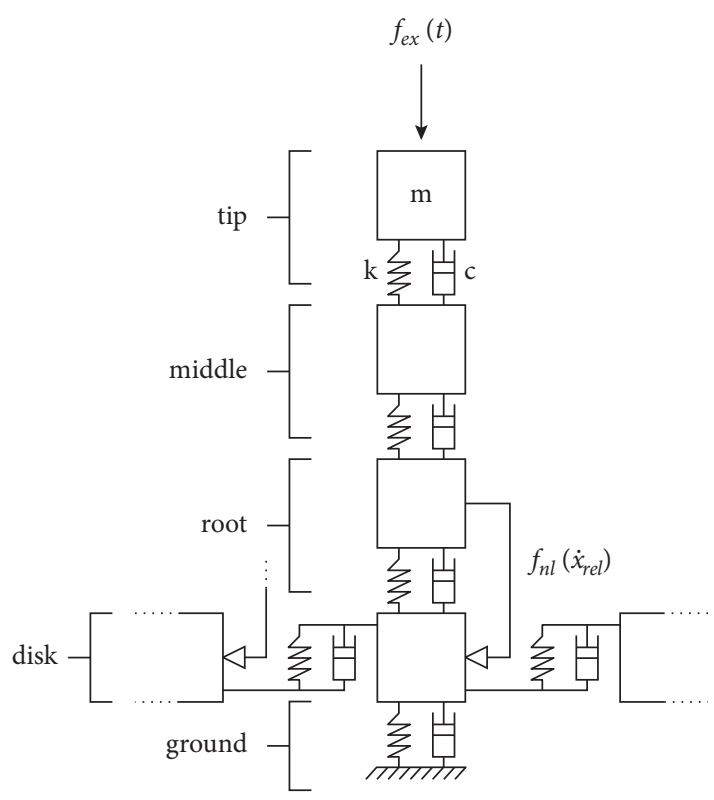

FIgURE 2: Fundamental sector of the model provided by reference [40].

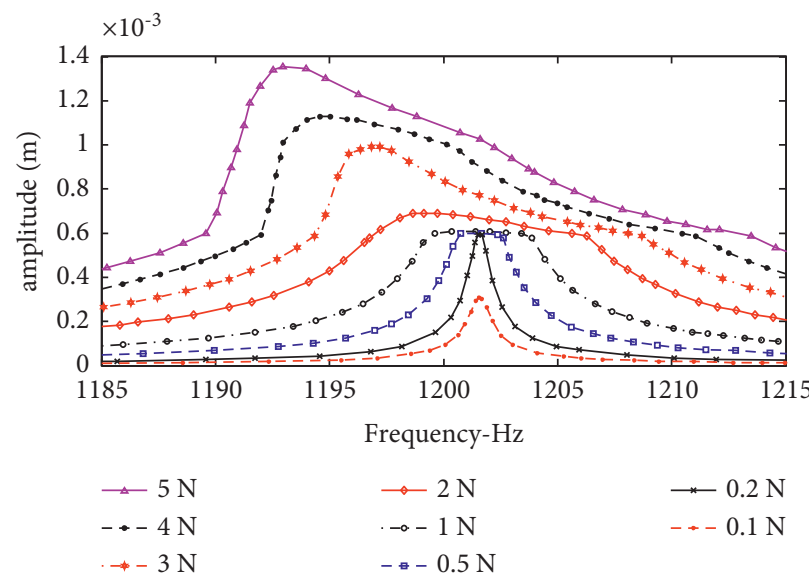

FIGURE 3: Frequency response function of reference [40]. Continuous lines are for reference [40] and circle point is regenerated of this paper for different excitation forces.

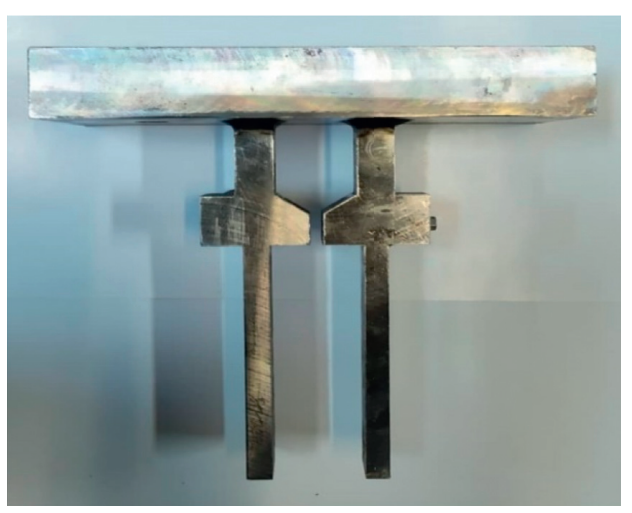

(a)

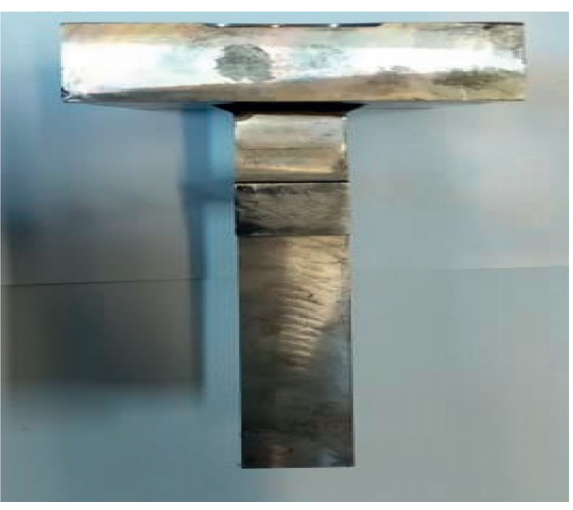

(b)

Figure 4: Laboratory sample-experimental test: (a) direct view; (b) side view. 


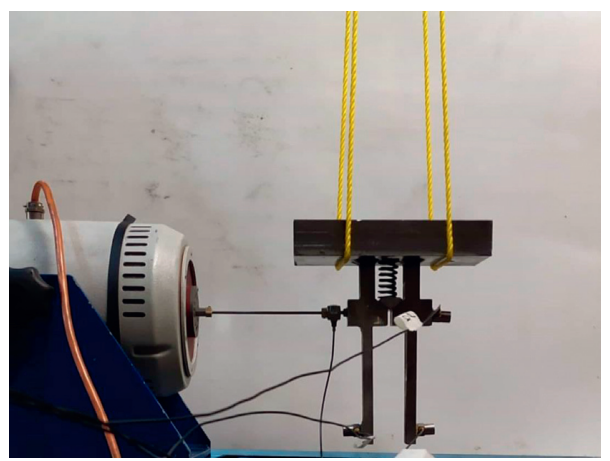

FIGURE 5: Experimental setup suspension configuration and sensor attachments configuration.
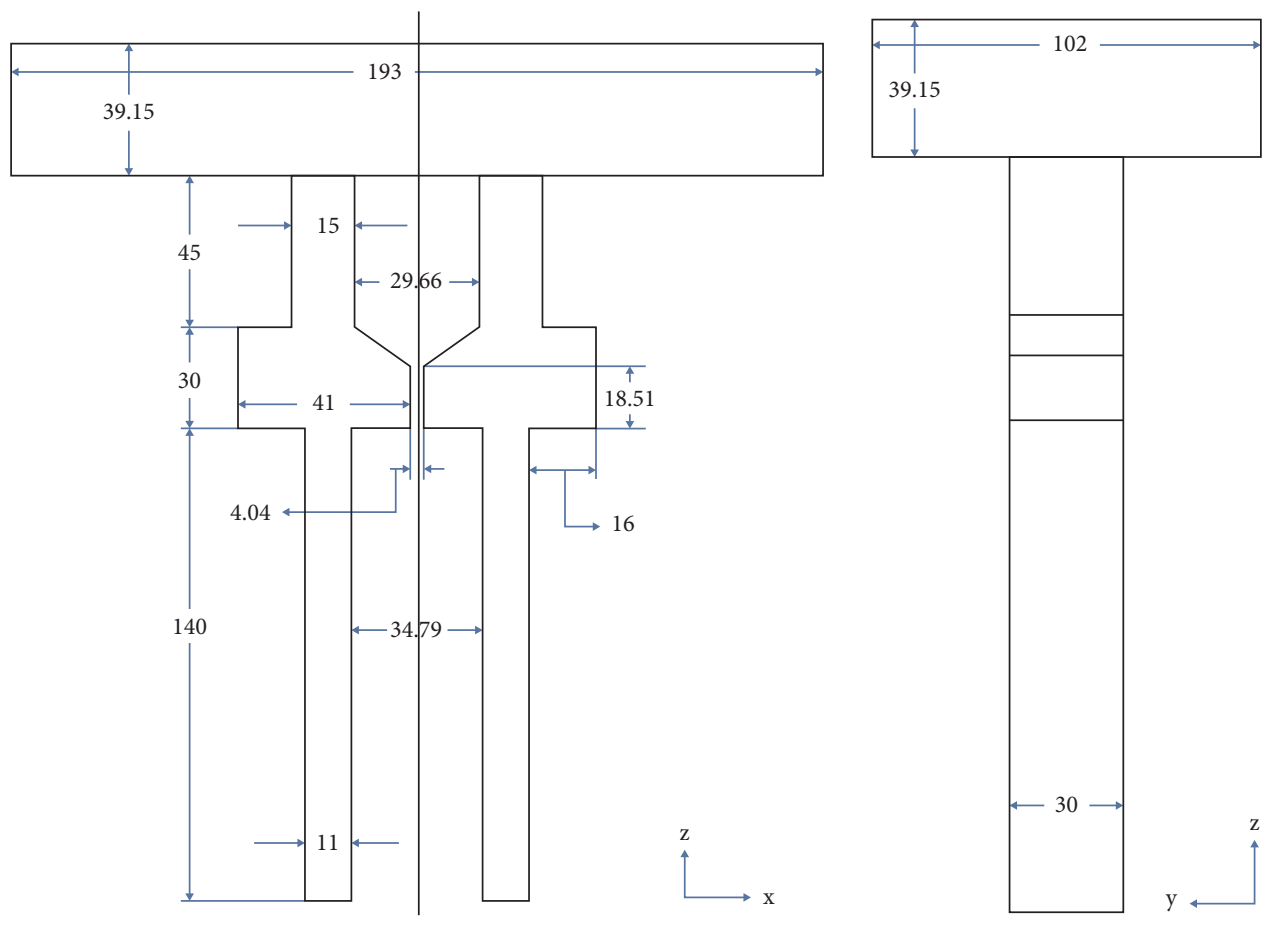

Figure 6: Laboratory sample size in two side views.

frequency of the structure and to identify the nonlinear effect of contact between under-platform damper and blade; sine sweep excitation in the first natural frequency range is used. Three accelerometers are used to measure the responses of the system to signal excitation in the structure (according to Figure 7). The frequency response function (FRF) of the linear system (without an under-platform damper) is shown in Figure 8. Two natural frequencies and related damping ratios from numerical analysis are shown in Table 1 . That is appropriately consistent with experimental results.

This analysis is done to capture linear frequencies and compare them with nonlinear ones and investigate the effect of nonlinear coupling on the natural frequency of the system (this comparison is shown in Table 2).

3.2. Nonlinear Analysis. In the nonlinear setup, a wedge and spring simulate an under-platform damper in a real turbine blade. In this model, the spring force simulates the centrifugal force. To restrict nonlinearity just to friction on the surface, the stiffness of spring is measured in the dynamic range of displacement to satisfy the linearity of spring in the dynamic range of measurement (Figure 9).

In Figures 7 and 10, FRF and coherence function of nonlinear system at the tip of blade are shown, respectively:

As we can see from Figure 7, first natural frequency shifts to a higher frequency due to the nonlinearity of friction but nonlinearity has no effect on the second natural frequency, and it is the same as linear system ones. This is because the friction surfaces are not active in the second mode-unlike the first mode. In the second mode two blades deflections are the same and aligned and therefore relative displacement of under-platform damper in this mode is zero. To investigate this phenomenon, the first and second mode of the structure is analyzed by Finite element (Figure 11). The comparison of linear and nonlinear analysis also is shown in Figure 12. It is also seen that friction reduces the vibration amplitude of the system in the first mode. The coherence function confirms 


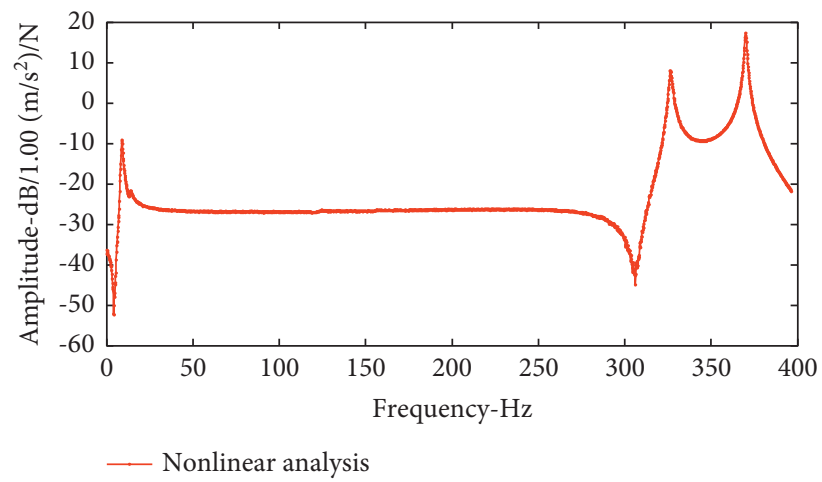

FIGURE 7: Frequency response function (FRF) of tip accelerometer for two elastic vibration modes (random excitation force $10.8 \mathrm{~N}$ ).

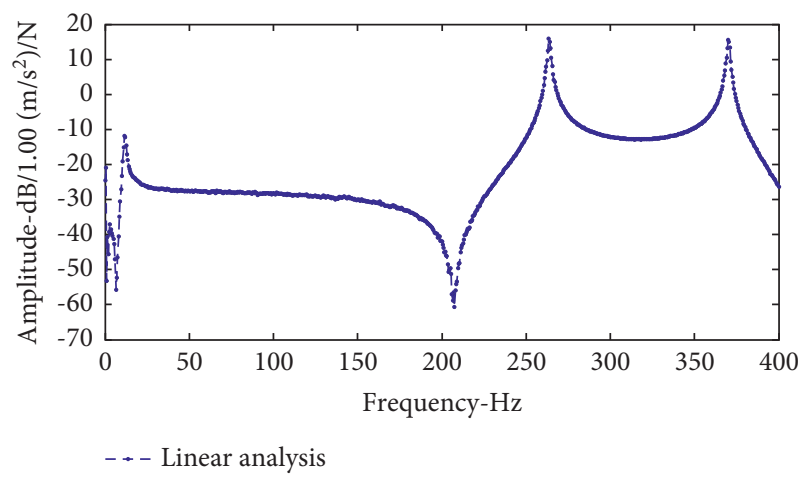

FIGURE 8: Frequency response function of linear system (random excitation force $10.8 \mathrm{~N}$ ).

TABLE 1: Identified parameters of the reference model [2] in the experimental model.

\begin{tabular}{cccccc}
\hline$N$ & $M$ & $C_{p}$ & $C_{q}$ & $K_{N}$ & $C_{k}$ \\
\hline 1 & 1 & $13.5 \times 10^{+3}$ & 0.18 & 78.5 & 0.56 \\
\hline
\end{tabular}

TABLE 2: Two natural frequencies and related damping ratio from numerical analysis.

\begin{tabular}{lc}
\hline$\omega_{n 1(\mathrm{~Hz})}$ & 264 \\
$\omega_{n 2(\mathrm{~Hz})}$ & 371 \\
$\eta_{n 1}$ & 0.0028 \\
$\eta_{n 2}$ & 0.001865 \\
\hline
\end{tabular}

that the system response is due to the system input and the noise to signal ratio is negligible, especially in the resonance range (Figure 10). At the following of the analysis, the system's frequency response at different excitation forces in the frequency range of the first vibration mode of the system is investigated.

The effect of excitation force level is shown in Figure 13, in this analysis FRF of the tip accelerometer for three different levels of excitation are showed:

In Figure 13, as the excitation force increases, the first frequency of the system shifts downwards. Since the stiffness of the structure decreases with increasing slip, it causes a decrease in the frequency of the structure and limit case, which tends to linear system response. Therefore, nonlinear friction contact is softening type.
In the following, the analytical results with Mohamadali model [2] that consider coupling effect of tangential direction on normal ones compare with experimental results. In this model friction and normal force are expressed by (3) and (4) respectively. In the normal force relation, the effect of tangential displacement on the variation of force is considered (coupling effect) that innovation of this paper is investigation this coupling effect on the response of turbine blade and exactly prediction response with considering this coupling that exists in physic of contact surfaces [17].

To find the frequency response of the system under test, the contact surface parameters must first be identified by the test results, which are extracted according to Table 1 . In this practice, the parameters of the contact model $(n, m$, $C_{P}, C_{Q}, C_{K}$ and $\left.K_{N}\right)$ are identified by minimizing the response between measure and prediction $\left|a_{\text {measured }}-a_{\text {prediction }}\right|$ with same excitation force, where $a_{\text {measured }}$ and $a_{\text {prediction }}$ are displacement measurement form experimental and model prediction, respectively. Detailed information about the method of parameter identification is presented in Ref [47].

By identifying these parameters, it is observed that this model can accurately predict the frequency response of the system at different forces by considering the effect of tangential displacement on the normal force.

As we can see from Figure 14, this model considers coupling effect between normal and tangential directions that is common phenomena in contact surface and recently confirm by reference [17], predict frequency response function more exactly related to the previous models that used in the turbine-blade field to simulated contact force of surface.

To more investigate the effect of tangential displacement on the normal force and as a result, the exact location of the resonance frequency and the resonance amplitude, the frequency response of the system at the excitation force of $6.37 \mathrm{~N}$ in both coupling and noncoupling modes have been compared with the test results (Figure 15). In the Mohammadali model, coupling effect is controlled by equation (4).

As can be seen in Figure 15, by ignoring the coupling effect (like other models used to simulate nonlinear force in the contact surface of turbine blade field) the frequency 


\begin{tabular}{|cc|}
\hline Displacement & Force \\
\hline$(\mathrm{mm})$ & $(\mathrm{N})$ \\
\hline 88.0 & 10.120 \\
\hline 84.9 & 53.980 \\
\hline 79.7 & 131.580 \\
\hline 76.8 & 175.450 \\
\hline 73.7 & 209.190 \\
\hline 71.4 & 229.430 \\
\hline 65.7 & 316.576 \\
\hline 53.5 & 482.020 \\
\hline 43.52 & 617.380 \\
\hline 30.32 & 796.420 \\
\hline 28.68 & 818.640 \\
\hline
\end{tabular}

(a)

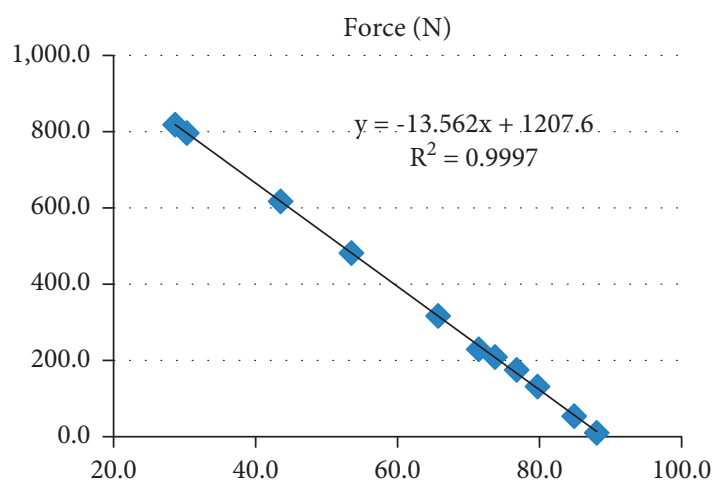

(b)

Figure 9: (a) Displacement and force of spring; (b) stiffness calculation.

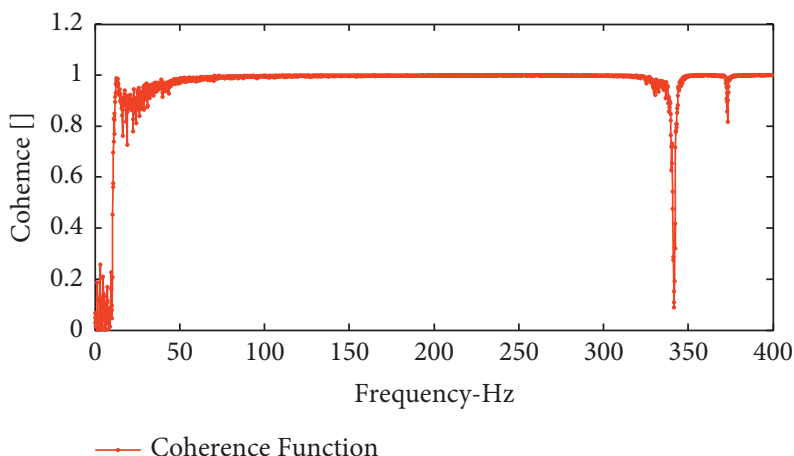

FIgURE 10: Coherence function of tip accelerometer in two modes vibration range (random excitation force $10.8 \mathrm{~N}$ ).

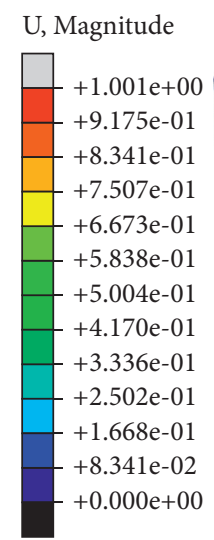

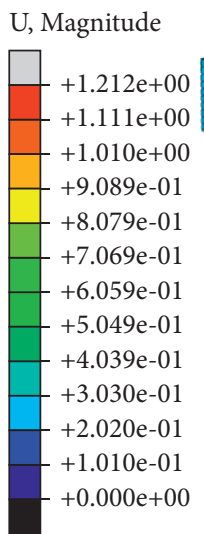

(a)

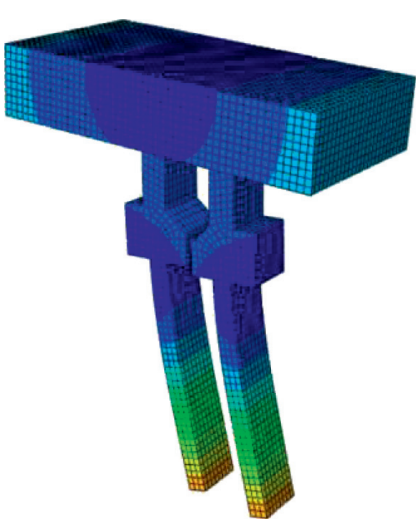

(b)

Figure 11: First and second vibration mode of experimental set-up-Finite element analysis. (a) First mode; (b) second mode.

response function prediction is associated with an error; especially this error is significant in determining the exact resonance amplitude. Considering this phenomenon leads to accurate determination of the amplitude and frequency of resonance. That numerical investigation also confirms this effect (amplitudes are expressed in linear numerically): 


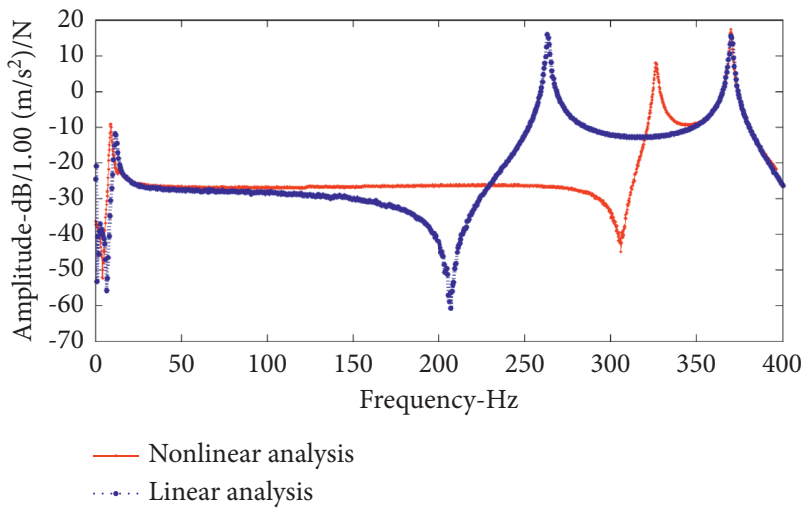

FIGURE 12: The comparison of linear and nonlinear analysis (random excitation force is $10.8 \mathrm{~N}$ ).

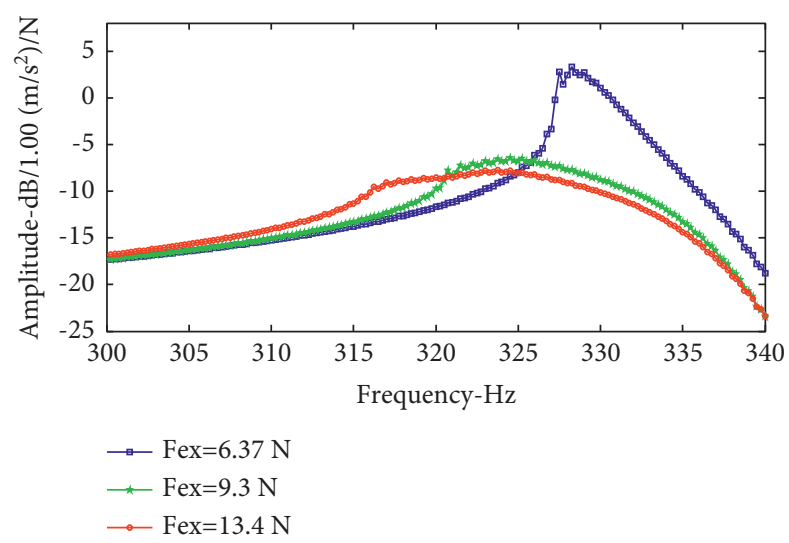

FIGURE 13: Frequency response function at different excitation force levels.

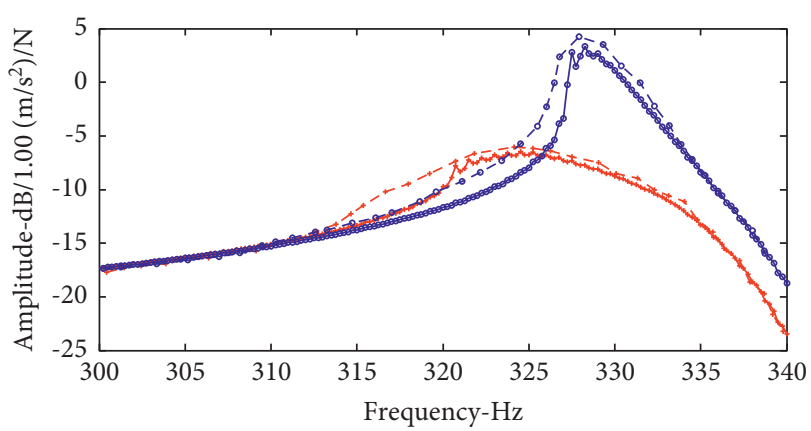

\begin{tabular}{ll}
$\longrightarrow$ Fex $=6.37 \mathrm{~N}$ Expermintal & - Fex $=9.3 \mathrm{~N}$ Expermintal \\
\hdashline-+ Fex $=6.37 \mathrm{~N}$ Model & $\rightarrow-F e x=F e x=9.3 \mathrm{~N}$ Model
\end{tabular}

Figure 14: Comparison of the result of experimental test and prediction response of the model with considering coupling effect. Continuous lines are experimental results, and dashed lines are theoretical model.

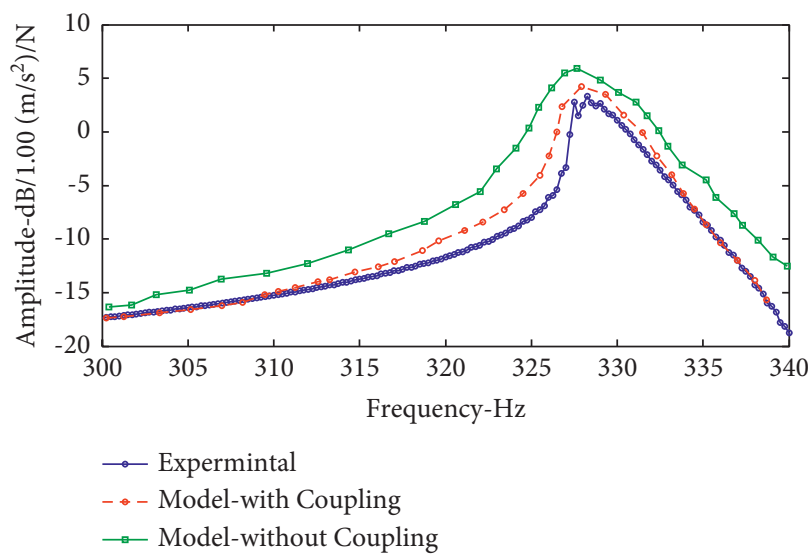

FIGURE 15: Investigation coupling effect on the frequency response of the system at the excitation force of $6.37 \mathrm{~N}$ (displacement unit of the meter).

$$
\begin{aligned}
& \frac{\max (a m p)_{\exp }-\max (a m p)_{W . c o u p l i n g}}{\max (a m p)_{\exp }}=\frac{0.027-0.0265}{0.027}=0.02, \\
& \frac{\max (a m p)_{\exp }-\max (a m p)_{\text {Without.coupling }}}{\max (a m p)_{\exp }}=\frac{0.027-0.03}{0.027}=0.11 .
\end{aligned}
$$


As can be seen in equations (9) and (10), ignoring the coupling effect in the theoretical model causes the prediction of the vibrational resonance amplitude to be associated with an error of $11 \%$ while with consider coupling effect response of the system can be predicted only with $2 \%$ error. Therefore, to accurately predict the frequency response, it is necessary to consider the coupling between the tangential and normal directions.

\section{Conclusion}

Forced response vibration of nonlinear turbine blades has been investigated by the MHB-AFT method. To calculate nonlinear force and to solve the algebraic equations, the AFT method and Newton-Raphson method, respectively, along the continuation algorithm has been used. In this paper, the effect of tangential displacement on normal load variation on the frequency response function of turbine blade has been investigated. It was shown that the effect of coupling (tangential displacement on normal force) is considerable and avoiding it in the forced response vibration analysis cause error in the determination frequency of resonance and vibration amplitude. Forced responses of turbine blade results of the previous reference to investigate validation and effectiveness of the solution method applied in this paper have been regenerated. To investigate the coupling phenomenon, experimental analysis was performed, and it has been shown that considering this phenomenon causes experimental results to be predicted with high accuracy and ignore the coupling effect cause error to predict experimental results. To design dry friction optimally, considering the coupling effect is essential. Main benefits of using this contact model that consider coupling effect to simulate nonlinear force of under-platform damper are as follows:

(i) Providing an explicit nonlinear model that considers energy dissipation in tangential direction and coupling between tangential and normal direction, simultaneously, allowing for determination frequency response function in large scale turbine case, at a relatively low computational time.

(ii) Precise prediction of the frequency response function of turbine blades, considering contact surface lift-up and variation of normal force due to the tangential displacement related to other models that ignore this effect.

\section{Data Availability}

No data were used to support this study.

\section{Conflicts of Interest}

The authors declare that they have no conflicts of interest.

\section{References}

[1] E. Cigeroglu, Development of Microslip Friction Models and Forced Response Prediction Methods for Frictionally Constrained Turbine Blades, The Ohio State University, Columbus, OH, USA, 2007.
[2] H. Ahmadian and M. Mohammadali, "A distributed mechanical joint contact model with slip/slap coupling effects," Mechanical Systems and Signal Processing, vol. 80, pp. 206-223, 2016.

[3] E. Cigeroglu, N. An, and C.-H. Menq, "A microslip friction model with normal load variation induced by normal motion," Nonlinear Dynamics, vol. 50, no. 3, pp. 609-626, 2007.

[4] D. Li, C. Xu, M. Gola, and D. Botto, "Reduced-order modeling friction for line contact in a turbine blade damper system," In Nonlinear Dynamics and Control, pp. 197-205, Springer, Cham, Switzerland, 2020.

[5] B. D. Yang, M. L. Chu, and C. H. Menq, "Stick-slip-separation analysis and non-linear stiffness and damping characterization of friction contacts having variable normal load," Journal of Sound and Vibration, vol. 210, no. 4, pp. 461-481, 1998.

[6] J. H. Griffin, "Friction damping of resonant stresses in gas turbine engine airfoils," Journal of Engineering for Power, vol. 102, no. 2, pp. 329-333, 1980.

[7] C.-H. Menq, J. H. Griffin, and J. Bielak, "The forced response of shrouded fan stages," Journal of Vibration and Acoustics, vol. 108, no. 1, pp. 50-55, 1986.

[8] B. Yang, J. Chen, and C. Menq, "Prediction of resonant response of shrouded blades with 3D shroud constraint," in Proceedings of the. ASME 1998 International Gas Turbine and Aeroengine Congress and Exhibition, June 1999, Article ID V005T014A042.

[9] E. P. Petrov and D. J. Ewins, "Analysis of the worst mistuning patterns in bladed disk assemblies," Journal of Turbomachinery, vol. 125, no. 4, pp. 623-631, 2003.

[10] M. Afzal, I. Lopez Arteaga, and L. Kari, "An analytical calculation of the Jacobian matrix for 3D friction contact model applied to turbine blade shroud contact," Computers \& Structures, vol. 177, pp. 204-217, 2016.

[11] N. Guofang and R. Xingmin, "Nonlinear dynamic analysis of shrouded turbine blade of aero-engine subjected to combination effect of impact and friction," in Proceedings of the. Computer Modelling and Simulation (UKSim), 2011 UkSim 13th International Conference on, pp. 212-217, IEEE, Cambridge, UK, March 2011.

[12] X. Guo, J. Zeng, H. Ma, C. Zhao, L. Qu, and B. Wen, "Dynamic characteristics of a shrouded blade with impact and friction," Frontiers of Mechanical Engineering, vol. 15, pp. 1-18, 2020.

[13] W. Gu, Z. Xu, and L. Qiang, "Forced response of shrouded blades with intermittent dry friction force," in Proceedings of the. ASME Turbo Expo 2008: Power for Land, Sea, and Air, pp. 501-508, American Society of Mechanical Engineers (ASME), Berlin, Germany, June 2008.

[14] H. Ma, F. Xie, H. Nai, and B. Wen, "Vibration characteristics analysis of rotating shrouded blades with impacts," Journal of Sound and Vibration, vol. 378, pp. 92-108, 2016.

[15] S. Chu, D. Cao, S. Sun, J. Pan, and L. Wang, "Impact vibration characteristics of a shrouded blade with asymmetric gaps under wake flow excitations," Nonlinear Dynamics, vol. 72, no. 3, pp. 539-554, 2013.

[16] P. Pennacchi, S. Chatterton, N. Bachschmid, E. Pesatori, and G. Turozzi, "A model to study the reduction of turbine blade vibration using the snubbing mechanism," Mechanical Systems and Signal Processing, vol. 25, no. 4, pp. 1260-1275, 2011.

[17] H. Jamshidi and H. Ahmadian, "A modified rough interface model considering shear and normal elastic deformation couplings," International Journal of Solids and Structures, vol. 203, pp. 57-72, 2020. 
[18] J. Den Hartog, "Forced vibrations with combined Coulomb and viscous friction," Trans. ASME, vol. 53, no. 15, pp. 107$115,1931$.

[19] A. A. Ferri and A. C. Bindemann, "Damping and vibration of beams with various types of frictional support conditions," Journal of Vibration and Acoustics, vol. 114, no. 3, pp. 289296, 1992.

[20] J. J. Chen and C. H. Menq, "Periodic response of blades having three-dimensional nonlinear shroud constraints," Journal of Engineering for Gas Turbines \& Power, vol. 123, no. 4, pp. 901-909, 2001.

[21] B. D. Yang and C. H. Menq, "Characterization of 3D contact kinematics and prediction of resonant response of structures having 3D frictional constraint," Journal of Sound and Vibration, vol. 217, no. 5, pp. 909-925, 1998.

[22] G. Nan, J. Lou, C. Song, and M. Tang, "A new approach for solving rub-impact dynamic characteristics of shrouded blades based on macroslip friction model," Shock and Vibration, vol. 2020, Article ID 8147143, 9 pages, 2020.

[23] T. Butlin, G. Spelman, P. Ghaderi, W. J. B. Midgley, and R. Umehara, "Predicting response bounds for frictiondamped gas turbine blades with uncertain friction coupling," Journal of Sound and Vibration, vol. 440, pp. 399$411,2019$.

[24] K. Y. Sanliturk, D. J. Ewins, and A. B. Stanbridge, "Underplatform dampers for turbine blades: theoretical modeling, analysis, and comparison with experimental data," Journal of Engineering for Gas Turbines \& Power, vol. 123, no. 4, pp. 919-929, 2001.

[25] S. Filippi, A. Akay, and M. M. Gola, "Measurement of tangential contact hysteresis during microslip," Journal of Tribology, vol. 126, no. 3, pp. 482-489, 2004.

[26] L. Hou, Y. Chen, Y. Fu, H. Chen, Z. Lu, and Z. Liu, “Application of the HB-AFT method to the primary resonance analysis of a dual-rotor system," Nonlinear Dynamics, vol. 88, no. 4, pp. 2531-2551, 2017.

[27] L. Hou, H. Chen, Y. Chen, K. Lu, and Z. Liu, "Bifurcation and stability analysis of a nonlinear rotor system subjected to constant excitation and rub-impact," Mechanical Systems and Signal Processing, vol. 125, pp. 65-78, 2019.

[28] C. Sun, Y. Chen, and L. Hou, "Steady-state response characteristics of a dual-rotor system induced by rub-impact," Nonlinear Dynamics, vol. 86, no. 1, pp. 91-105, 2016.

[29] C. Gastaldi, T. M. Berruti, and M. M. Gola, "A novel test rig for friction parameters measurement on underplatform dampers," International Journal of Solids and Structures, vol. 185-186, pp. 170-181, 2020.

[30] D. Botto, M. Umer, C. Gastaldi, and M. M. Gola, “An experimental investigation of the dynamic of a blade with two under-platform dampers," in Proceedings of the. Turbo Expo: Power for Land, Sea, and Air, June 2017, Article ID V07BT35A029.

[31] E. Ferhatoglu and S. Zucca, "Determination of periodic response limits among multiple solutions for mechanical systems with wedge dampers," Journal of Sound and Vibration, vol. 494, Article ID 115900, 2021.

[32] E. Ferhatoglu and S. Zucca, "On the non-uniqueness of friction forces and the systematic computation of dynamic response boundaries for turbine bladed disks with contacts," Mechanical Systems and Signal Processing, vol. 160, Article ID 107917, 2021.

[33] Y. Wu, Y. Fan, L. Li, and Z. Zhao, "On the performance of wavy dry friction and piezoelectric hybrid flexible dampers,"
Journal of Engineering for Gas Turbines \& Power, vol. 143, no. 12, Article ID 121010, 2021.

[34] H. You, Forced vibration characteristics of bladed disc assemblies, University of London, PhD thesis, 1995.

[35] L. Panning, W. Sextro, and K. Popp, "Optimization of the contact geometry between turbine blades and underplatform dampers with respect to friction damping," in Proceedings of the Turbo Expo: Power for Land, Sea, and Air, pp. 991-1002, Berlin, Germany, June 2002.

[36] M. Krack and J. Gross, Harmonic Balance for Nonlinear Vibration Problems, Springer, Berlin, Germany, 2019.

[37] C. Joannin, B. Chouvion, F. Thouverez, M. Mbaye, and J.-P. Ousty, "Nonlinear modal analysis of mistuned periodic structures subjected to dry friction," Structures and Dynamics, vol. 7B, 2015.

[38] S. Zucca, C. M. Firrone, and M. Gola, "Modeling underplatform dampers for turbine blades: a refined approach in the frequency domain," Journal of Vibration and Control, vol. 19, no. 7, pp. 1087-1102, 2013.

[39] E. Cigeroglu, W. Lu, and C.-H. Menq, "One-dimensional dynamic microslip friction model," Journal of Sound and Vibration, vol. 292, no. 3, pp. 881-898, 2006.

[40] C. Joannin, B. Chouvion, F. Thouverez, M. Mbaye, and J.-P. Ousty, "Nonlinear modal analysis of mistuned periodic structures subjected to dry friction," Journal of Engineering for Gas Turbines \& Power, vol. 138, no. 7, 2016.

[41] E. P. Petrov, "Method for direct parametric analysis of nonlinear forced response of bladed disks with friction contact interfaces," Journal of Turbomachinery, vol. 126, no. 4, pp. 654-662, 2004.

[42] M. Afzal, On Efficient and Adaptive Modelling of Friction Damping in Bladed Disks, KTH Royal Institute of Technology, Stockholm, Sweden, 2017.

[43] C. Schwingshackl, E. Petrov, and D. Ewins, "Effects of contact interface parameters on vibration of turbine bladed disks with underplatform dampers," in Proceedings of the ASME 2011 Turbo Expo: Turbine Technical Conference and Exposition, pp. 867-876, American Society of Mechanical Engineers Digital Collection, Vancouver, British Columbia, June 2012.

[44] A. Bernadez, Nonlinear Normal Modes in Mechanical Systems: Concept and Computation with Numerical Continuation, University of Madrid, Madrid, Spain, 2016.

[45] C. M. Firrone, "Measurement of the kinematics of two underplatform dampers with different geometry and comparison with numerical simulation," Journal of Sound and Vibration, vol. 323, no. 1-2, pp. 313-333, 2009.

[46] L. Pesaresi, L. Salles, A. Jones, J. S. Green, and C. W. Schwingshackl, "Modelling the nonlinear behaviour of an underplatform damper test rig for turbine applications," Mechanical Systems and Signal Processing, vol. 85, pp. 662679, 2017.

[47] M. Mohammadali and H. Ahmadian, "Efficient model order reduction for dynamic systems with local nonlinearities," Journal of Sound and Vibration, vol. 333, no. 6, pp. 1754-1766, 2014. 